\title{
A New and Simple Predictive Formula for Non-Sentinel Lymph Node Metastasis in Breast Cancer Patients with Positive Sentinel Lymph Nodes, and Validation of 3 Different Nomograms in Turkish Breast Cancer Patients
}

\author{
Levent Yeniay Erdem Carti Can Karaca Osman Zekioglu \\ Ulkem Yararbas Rasih Yilmaz Murat Kapkac
}

Ege University School of Medicine, Izmir, Turkey

\section{Keywords}

Sentinel lymph node biopsy - MSKCC nomogram .

Tenon score · Turkish nomogram · Non-sentinel lymph node metastasis

\section{Summary}

Background: Nomogram accuracies for predicting nonsentinel lymph node (SLN) involvement vary between different patient populations. Our aim is to put these nomograms to test on our patient population and determine our individual predictive parameters affecting SLN and non-SLN involvement. Patients and Methods: Data from 932 patients was analyzed. Nomogram values were calculated for each patient utilizing MSKCC, Tenon, and MHDF models. Moreover, using our own patient- and tumor-depended parameters, we established a unique predictivity formula for SLN and non-SLN involvement. Results: The calculated area under the curve (AUC) values for MSKCC, Tenon, and MHDF models were 0.727 (95\% confidence interval (Cl) $0.64-0.8), 0.665$ (95\% Cl 0.59-0.73), and $0.696(95 \% \mathrm{Cl} 0.59-0.79)$, respectively. Cerb-2 positivity $(p=0.004)$ and size of the metastasis in the lymph node ( $p=0.006$ ) were found to correlate with non-SLN involvement in our study group. The AUC value of the predictivity formula established using these parameters was 0.722 (95\% Cl 0.63-0.81). Conclusion: The most accurate nomogram for our patient group was the MSKCC nomogram. Our unique predictivity formula proved to be as equally effective and competent as the MSKCC nomogram. However, similar to other nomograms, our predictivity formula requires future validation studies.

\section{Schlüsselwörter}

Sentinel-Lymphknotenbiopsie - MSKCC-Nomogramm • Tenon-Score - Türkisches Nomogramm . Nicht-Sentinel-Lymphknotenmetastasen

\section{Zusammenfassung}

Hintergrund: Die Genauigkeit von Nomogrammen zur Vorhersage einer Beteiligung von Nicht-Sentinel-Lymphknoten (SLK) schwankt zwischen verschiedenen Patientenpopulationen. Ziel unserer Studie war es, die entsprechenden Nomogramme an unserer Patientenpopulation zu testen und individuelle prädiktive Parameter, die Auswirkungen auf die Beteiligung von SLK und NichtSLK haben, zu bestimmen. Patienten und Methoden: Die Daten von 932 Patienten wurden analysiert. Die Nomogrammwerte für jeden Patienten wurden unter Einsatz der Modelle MSKCC, Tenon und MHDF berechnet. Unter Einbeziehung unserer eigenen patienten- und tumorabhängigen Parameter wurde desweiteren eine spezifische Prädiktivitätsformel zur Vorhersage der SLK- bzw. NichtSLK-Beteiligung entwickelt. Ergebnisse: Die berechneten AUC (area under the curve)-Werte für MSKCC, Tenon bzw. MHDF waren 0,727 (95\%-Konfidenzinterval (KI) 0,64-0,8), 0,665 (95\%-KI 0,59-0,73) bzw. 0,696 (95\%-KI $0,59-0,79)$. Cerb-2-Positivität $(p=0,004)$ und Größe der Lymphknotenmetastasen $(p=0,006)$ korrelierten in unserer Studie mit Nicht-SLK-Beteiligung. Der mittels dieser Parameter etablierte AUC-Wert der Prädiktivitätsformel war 0,722 (95\%-KI 0,63-0,81). Schlussfolgerung: Das akkurateste Nomogramm für unsere Patientengruppe war das MSKCC-Nomogramm. Unsere neu erstellte Prädiktivitätsformel erwies sich als ebenso effektiv und kompetent wie das MSKCC-Nomogramm. Wie auch mit anderen Nomogrammen bedarf es jedoch zukünftiger Validierungsstudien, um dies zu bestätigen.

Dr. Levent Yeniay

School of Medicine

Ege Universitesi Tip Fakultesi Genel Cerrahi AD

3. KAt Sekreterlik Bornova, 35100 Izmir, Turkey

levent.yeniay@ege.edu.tr 


\section{Introduction}

Breast cancer is a major public health concern for which surgery is the first treatment step. Surgery establishes local control of the disease and yields critical prognostic data such as lymph node status and involvement [1]. Historically, axillary dissection (AD) was the sole procedure used to achieve this goal; however, its associated morbidities, first and foremost lymph edema [2, 3], triggered the search for less invasive methods. The technique of sentinel lymph node biopsy (SLNB) emerged as a breakthrough result from these research efforts [4]. The efficacy and reliability of SLNB has been proven in many randomized controlled studies, and it has become a strong alternative to AD in eligible patients. Many case series have shown that SLNB protects nearly half of all patients from unnecessary AD. While AD still remains the gold standard for patients with positive SLN, only 30-50\% [5-7] of these have positive non-SLN (NSLN). Thus, numerous studies aim to establish a scientific basis of foreseeing the metastatic status of the NSLN by evaluating numerous parameters belonging to patients and tumors. The Memorial Sloan Kettering Cancer Center (MSKCC, 2003) [8], Tenon Hospital (2005) [9], Cambridge University (2008) [10], Stanford University (2008) [11], and Federation of Breast Disease Associations of Turkey (MHDF, 2010) [12] all have their own scoring system based on the prognostic data derived from their studies. However, the accuracy of these nomograms varies among health centers and patient populations. We aimed to evaluate the accuracy of these scoring systems, and establish a new system based on the prognostic values found in our own patient population.

\section{Patients and Methods}

The records of patients who underwent SLNB between June 2003 and June 2011 were retrospectively analyzed. Patients with early-stage tumors and clinically negative axillae were enrolled in the study. Patients who underwent SLNB before neoadjuvant chemotherapy were excluded from the study. Several patient- and tumor-depended parameters were studied (patient age, tumor size, tumor site, histological type, luminal classification, number of resected SLN, number of para-SLN, presence and size of metastasis, receptor status, nuclear grade, modified Bloom-Richardson score (MBRS), radius of the metastatic foci, number of lymph nodes dissected during AD, presence of metastasis in the NSLN, and number of metastatic NSLN)

\section{$S L N B$}

The SLNB procedure was carried out as published elsewhere [11]. A radioactive colloid substance was applied via periareolar injection the day before the operation. Lymph nodes spotted by gamma probe as 'hot nodes' were resected assuming they were SLN. Background nuclear activity was re-tested to make sure that all 'hot nodes' were removed. Moreover, the axilla was double-checked by inspection and palpation to avoid leaving any suspicious nodes behind. Suspicious nodes detected in this second check were also removed and named para-SLN. Frozen section analyses of the SLN and para-SLN were obtained perioperatively.
AD was performed for level I and II lymph nodes if any macro- or micrometastases were reported from the frozen section analysis. In the event of false negativity, AD was performed in a second operation.

\section{Pathologic Evaluation}

Frozen section analysis of the SLN was performed in 2 phases: intraoperatively and postoperatively. For intraoperative analysis, both touch imprint and frozen section were employed. For frozen section analysis, nodes were evaluated according to their sizes. Nodes smaller than $3 \mathrm{~mm}$ in radius were evaluated by a single section, nodes between $3-10 \mathrm{~mm}$ in radius by 2 sections, and nodes larger than $10 \mathrm{~mm}$ in radius by multiple 2-mm thick sections. The remainders of the SLN were formalin-fixed, paraffin-embedded, and sectioned in $50-\mu \mathrm{m}$ thick pieces all along the tissue. 2 sections from every $50-\mu \mathrm{m}$ thick piece were collected for staining with hematoxylin and eosin (HE) and cytokeratin (Clone AE1/AE3; Dako, Glostrup, Denmark). HE staining was performed for histological evaluation, and immunohistochemistry using the cytokeratin antibody was used for lymph nodes found to be negative during HE staining. SLN were diagnosed as positive when 1 of the 3 evaluation methods (frozen section, HE-stained section, immunohistochemistry) proved to be so. Lymph node metastases were classified into 3 groups according to size: macrometastases (>2 mm), micrometastases $(0.2-2 \mathrm{~mm})$, and submicrometastases (isolated tumor cells, $<0.2 \mathrm{~mm}$ ). Patients with macro- or micrometastases in their SLN underwent complementary AD. Isolated tumor cells were not considered as metastatic nodes and did not constitute an indication for complementary AD. The same pathologic evaluation was carried out for para-SLN.

\section{Nomograms}

MSKCC nomogram values and Tenon, MHDF, and MF-08 scores were calculated for each patient. MSKCC nomogram values were obtained from the website nomograms.mskcc.org/breast/ for the SLN (MSKCC nomogram 1) and NSLN (MSKCC nomogram 2). Tenon scores were calculated based on the scoring method described in the original article. MHDF and MF-08 scores were also calculated using the predictivity formulas in the original articles.

\section{Statistical Analysis}

The relationship between parameters and SLN/NSLN was assessed with univariate and multivariate analyses. The Statistics Package for Social Sciences (SPSS) version 16.0 (SPSS Inc., Chicago, IL, USA) was used for all statistical analyses. Tests included chi square, analysis of variance (ANOVA), receiver-operating characteristic (ROC) curve, Fisher's exact test, Mann-Whitney $\mathrm{U}$ test, and logistic regression.

\section{Results}

Including bilateral intervention in 10 patients, 995 successful SLNB procedures were carried out for 985 patients during the mentioned time period. 53 patients with non-invasive tumors (ductal or lobular carcinoma in situ and Paget's disease) and 10 patients with false-negative SLNB results were excluded from the study. All patients except for 1 were female. The median age was $51.9 \pm 11.6$ years (range $19-85$ years). A total of 2,565 SLN were excised. A median number of 2.75 (1-10) SLN were excised for each patient. The median tumor size was $18 \pm 8.9 \mathrm{~mm}$ (range $0.1-50 \mathrm{~mm}$ ). The radius of the SLN metastases was clearly defined in the records of only 164 patients, with a median value of $4.6 \pm 4.7 \mathrm{~mm}$ (range $0.1-22 \mathrm{~mm}$ ). The MBRS was recorded for only 394 patients who under- 
Table 1. Descriptive statistics of the patient population

\begin{tabular}{|c|c|c|c|}
\hline \multirow[t]{2}{*}{ Characteristics } & \multicolumn{3}{|l|}{ Patients, n (\%) } \\
\hline & $\begin{array}{l}\text { SLN-negative } \\
\text { (benign and } \\
\text { submicrometastasis) }\end{array}$ & $\begin{array}{l}\text { SLN-positive } \\
\text { (macro- } / \\
\text { micrometastasis) }\end{array}$ & Total \\
\hline Age, years & & & 932 \\
\hline$\leq 50$ & 311 (46.6) & $141(53.0)$ & \\
\hline$>50$ & $355(53.3)$ & $125(46.9)$ & \\
\hline Tumor size, $\mathrm{cm}$ & & & 913 \\
\hline$\leq 2$ & $488(74.5)$ & $142(55.0)$ & \\
\hline $2-5$ & $164(25.0)$ & $114(44.2)$ & \\
\hline$>5$ & $3(0.5)$ & $2(0.8)$ & \\
\hline Tumor type & & & 924 \\
\hline Ductal & $499(75.9)$ & $219(82.3)$ & \\
\hline Lobular & $34(5.1)$ & $14(5.2)$ & \\
\hline Other & $125(18.9)$ & $33(12.4)$ & \\
\hline Nuclear grade & & & 824 \\
\hline 1 & $120(21.0)$ & $24(9.5)$ & \\
\hline 2 & $378(66.1)$ & $184(73.0)$ & \\
\hline 3 & $74(12.9)$ & $44(17.5)$ & \\
\hline Lymphovascular invasion & & & 932 \\
\hline Yes & $47(7.0)$ & $116(43.7)$ & \\
\hline No & $620(92.9)$ & $149(56.2)$ & \\
\hline Estrogen receptor status & & & 928 \\
\hline Positive & $496(75.0)$ & $208(78.2)$ & \\
\hline Negative & $166(25.0)$ & $58(21.8)$ & \\
\hline Progesterone receptor status & & & 928 \\
\hline Positive & $430(65.0)$ & $195(73.3)$ & \\
\hline Negative & $232(35.0)$ & $71(26.7)$ & \\
\hline p 53 status & & & 709 \\
\hline Positive & $181(35.5)$ & $91(42.7)$ & \\
\hline Negative & $315(63.5)$ & $122(57.3)$ & \\
\hline $\mathrm{Ki}-67$ status & & & 741 \\
\hline Positive & $382(72.6)$ & $166(77.2)$ & \\
\hline Negative & $144(27.4)$ & $49(22.8)$ & \\
\hline Cerb-2 status & & & 930 \\
\hline Positive & $181(27.3)$ & $66(24.8)$ & \\
\hline Negative & $483(72.7)$ & $200(75.2)$ & \\
\hline MBRS & & & 394 \\
\hline 1 & $88(33.2)$ & $30(23.3)$ & \\
\hline 2 & $133(50.2)$ & $74(57.4)$ & \\
\hline 3 & 44 (16.6) & $25(19.4)$ & \\
\hline Number of positive SLN & & & 270 \\
\hline 1 & & $195(72.0)$ & \\
\hline 2 & & $51(18.8)$ & \\
\hline 3 & & $16(5.9)$ & \\
\hline 4 & & $6(2.2)$ & \\
\hline$\geq 5$ & & $2(0.7)$ & \\
\hline Number of negative SLN & & & 271 \\
\hline 0 & $415(62.7)$ & $99(36.5)$ & \\
\hline 1 & $123(18.5)$ & $79(29.2)$ & \\
\hline 2 & $58(8.7)$ & 39 (14.4) & \\
\hline 3 & 67 (10.1) & $29(10.7)$ & \\
\hline 4 & $57(8.6)$ & $13(4.8)$ & \\
\hline$\geq 5$ & & $12(4.4)$ & \\
\hline Proportion of positive SLN/total SLN & & & 244 \\
\hline$<0.5$ & & $74(30.3)$ & \\
\hline $0.5-<1$ & & $77(31.5)$ & \\
\hline 1 & & $93(38.1)$ & \\
\hline Luminal type & & & 928 \\
\hline A & $415(62.7)$ & $177(66.8)$ & \\
\hline B & $123(18.5)$ & $51(19.2)$ & \\
\hline $\operatorname{HER} 2(+)$ & $58(8.7)$ & $15(5.7)$ & \\
\hline Triple-negative & $67(10.1)$ & $22(8.3)$ & \\
\hline Multifocality & & & 931 \\
\hline Yes & $57(8.6)$ & $38(14.5)$ & \\
\hline No & $606(91.4)$ & $230(85.5)$ & \\
\hline Proportion & & & \\
\hline Benign/submicrometastasis & $625 / 36(67.1 / 3.8)$ & & $625 / 36$ \\
\hline Micrometastasis/macrometastasis & & 75/196 (8.0/21.0) & $75 / 196$ \\
\hline
\end{tabular}


Table 2. Summary of our ROC results

\begin{tabular}{lll}
\hline Nomogram & AUC & 95\% CI \\
\hline MSKCC for SLN metastases $(\mathrm{n}=736)$ & 0.744 & $0.70-0.78$ \\
MSKCC for non-SLN metastases $(\mathrm{n}=153)$ & 0.727 & $0.64-0.80$ \\
Tenon score $(\mathrm{n}=239)$ & 0.665 & $0.59-0.73$ \\
MHDF MF 08 predicted probability $(\mathrm{n}=143)$ & 0.696 & $0.759-0.79$ \\
Ege University predicted probability for SLN metastases $(\mathrm{n}=895)$ & 0.722 & $0.72-0.79$ \\
Ege University predicted probability for non-SLN metastases $(\mathrm{n}=143)$ & $0.63-0.81$ \\
\hline SLN = Sentinel lymph node; AUC = area under the curve; 95\% CI = 95\% confidence interval. \\
\hline
\end{tabular}

\begin{tabular}{lclc}
\hline Metastasis size & \multicolumn{3}{c}{ Patients, $\mathrm{n}(\%)$} \\
\cline { 2 - 4 } & total & negative NSLN & positive NSLN \\
\hline Micrometastasis & 61 & $52(85.2)$ & $9(14.8)$ \\
Macrometastasis & 183 & $92(50.3)$ & $91(49.7)$ \\
\hline NSLN = Non-sentinel lymph nodes. & \\
\hline
\end{tabular}

Table 3. Histopathologic evaluation of axillary lymph node dissection specimens $(\mathrm{p}=0)$ went surgery in the last 3 years. In evaluating the tumors for estrogen receptor, progesterone receptor, p53, and Ki-67 status, a $10 \%$ positivity for each parameter was accepted as positive. For Cerb-2, diffusely stained tumors (triple-positive) or tumors with positive fluorescent in situ hybridization (FISH) test (double-positive tumors) were accepted as positive. Complementary AD was performed in 244 patients, and the median number of dissected lymph nodes was $19 \pm 6.6$ (range 5-54). The median number of metastatic lymph nodes in the AD specimens of the patients with positive NSLN $(\mathrm{n}=100)$ was $3.2 \pm 4.4$ (range 1-35). Breast conserving surgery was applied in $71.4 \%$ of the patients. Definitive statistics of all patients are shown in table 1. Para-SLN were excised in 160 (26.8\%) patients. Evidence of metastasis was found in the para-SLN of 8 patients who had non-metastatic SLN. 2 additional patients had evidence of metastasis in their intramammarian lymph nodes but non-metastatic SLN. The false-negative rate was $1.1 \%$. The most common histological subtype was invasive ductal carcinoma $(71.3 \%)$. Other subtypes were invasive cribriform carcinoma $(4.2 \%)$, microinvasive ductal carcinoma in situ $(3.9 \%)$, invasive lobular carcinoma $(3.6 \%)$, and other rare subtypes $(16.8 \%)$. The median follow-up time was 34 months (range 1-93 months).

\section{Nomogram Results}

The area under the curve (AUC) values in the ROC curves established using the MSKCC nomograms 1 and 2, the Tenon model, and the MHDF MF-08 model were 0.744 (95\% confidence interval (CI) 0.7-0.78), 0.727 (95\% CI 0.64-0.8), 0.665 (95\% CI $0.59-0.73$ ), and 0.696 (95\% CI 0.59-0.79), respectively (table 2). In univariate analysis, a significant correlation was found between SLN positivity and tumor radius $(p=0)$, nuclear grade $(\mathrm{p}=0)$, invasive ductal carcinoma subtype $(\mathrm{p}=0)$, progesterone receptor positivity $(\mathrm{p}<0.017)$, and multifocality $(p<0.009)$. SLN invasion was present in 271 patients. Macrometastasis was observed in 196 (21\%) and micrometastasis in $75(8 \%)$ of these patients, and complementary AD was performed in 244 patients. 100 (40.9\%) patients had NSLN metastasis (table 3). In the complementary AD group,
NSLN positivity was found to be correlated with Cerb-2 positivity $(p=0.049)$, luminal A class tumor $(p=0.038)$, presence of lymphovascular invasion ( $\mathrm{p}=0$ ), number of positive SLN $(>3)(p=0)$, and radius of the SLN metastases $(p=0)$. In multivariate regression analysis of factors affecting SLN and NSLN positivity, tumor size $(\mathrm{p}=0)$, presence of lymphovascular invasion $(\mathrm{p}=0)$, and progesterone receptor positivity $(\mathrm{p}=0.012$ ) were found to be correlated with SLN positivity, while the radius of the metastatic foci in the SLN $(\mathrm{p}=0.006)$ and Cerb-2 positivity $(\mathrm{p}=0.004)$ were statistically correlated with NSLN positivity.

Using these results, we propose the following predictivity formula for SLN metastasis:

$\mathrm{p}=1 / 1+\exp (-2.414+0.048 \times$ tumor size $+0.533 \times$ proges terone receptor $+2.157 \times$ lymphovascular invasion);

and for NSLN metastasis:

$p=1 / 1+\exp (-2.721+1.696 \times$ Cerb $-2+0.117 \times$ metastasis radius).

The AUC values for SLN and NSLN in the ROC curves obtained by using these predictivity formulas were 0.759 (95\% CI 0.72-0.79) and 0.722 (95\% CI 0.63-0.81).

\section{Discussion}

SLNB was firmly established worldwide in routine practice by the end of the 1990s [2,13]. With the rapid increase in patient numbers, the SLNB technique proved to protect nearly half of all patients from unnecessary AD, and moreover it was shown that the SLN was the only involved node in up to $50-65 \%$ of the remaining patients. Setting out from these data, studies were launched worldwide to determine factors affecting SLN and NSLN involvement [14-16], which resulted in the establishment of a number of nomograms. The results of our study highlight these facts once again. The SLN involvement rate in our patients was $28.5 \%$; however, only $40.8 \%$ showed involvement of NSLN.

Today, the MSKCC nomogram [8], which was the first to be published, is most commonly used for predicting NSLN 
involvement. Van Zee et al. [8] demonstrated the accuracy of the nomogram, which was established based on statistical data derived from a retrospective study arm, by prospectively applying it to 373 patients whose breast cancer was detected through biopsy in the SLN. The nomogram accurately predicted NSLN involvement in these patients. According to the discriminative tests applied depending on these results, the AUC values of the ROC curves were 0.76 for SLN involvement and 0.77 for NSLN involvement. The MSKCC nomogram employs 9 patient-dependent variables. To this day, more than 30 studies [12, 17-21] have been published that have validated this most popular nomogram. Except for a few $[22,23]$, all studies reported ROC values higher than 0.7, proving the reliability of the MSKCC nomogram. In our patient population, the MSKCC nomogram also proved to be the most accurate (ROC values 0.744 for SLN and 0.727 for NSLN involvement).

In 2005, Barranger et al. [9] published the Tenon scoring model. In a prospective study covering 337 patients, presence of macrometastases, tumor radius, and ratio of positive SLN over the total number of resected nodes were found to be correlated with NSLN involvement. After applying points between 1 and 3, parameters are added up to define a likelihood score with a maximum of 7 points. Patients with a score of 3.5 or more were reported to have a likelihood ratio for negative NSLN of $97.3 \%$ (95\% CI 20.5-90.0). According to the authors, the weakness of this scoring system is that it does not take the size of nodal metastasis and extranodal dissemination into account [9]. Studies that validated the Tenon score, reported ROC values of 0.75 [17], 0.82 [24], and 0.582 [12]. The third study, which reported the lowest value, was carried out by Gur et al. [12] on a Turkish patient population. Similarly, we also derived the lowest ROC values from the application of the Tenon scoring system (ROC 0.665) with our patient population, suggesting a racial similarity. In a number of studies with a low-risk (micrometastases) patient setting, the Tenon model was found to be more accurate and useful than the MSKCC nomogram [23].

In the study by Gur et al. [12] in 2010, the authors reviewed and applied the MSKCC, Stanford, Cambridge, and Tenon models to their patient population, and proposed a genuine predictivity formula fitting this population. The ROC values for the 4 models from this first Turkish nomogram study on a population showing racial similarities to our patient population were $0.705,0.711,0.730$, and 0.582 , respectively. Radius of the metastatic foci, ratio of positive SLN over total number of resected nodes, and lymphovascular invasion correlated with likelihood of NSLN involvement. Among these variables, lymphovascular invasion had the highest odds ratio. The discriminative value of the proposed predictivity formula was 0.802 . In our study, the ROC value of the MHDF model (0.696) was placed right below the confidence border for any given model $(0.7)$. What could be the reason for this incoherence? There are some differences between the population studied by Gur et al. [12] and our patient population. The most important differences include a lower mean tumor radius (25 vs. $18.6 \mathrm{~mm}$ ), fewer lobular subtype tumors (12.9 vs. $5.2 \%$ ), a higher incidence of micrometastasis (17.6 vs. $27.6 \%$ ), and a lower incidence of lymphovascular invasion (53.8 vs. $43.7 \%$ ) in our study. Another probable explanation for these differences is that, as a multicentric study, Gur et al.'s [12] pathologic evaluation could have lacked uniformity. In addition, the number of patients was substantially higher in Gur et al.'s study than in our study ( $n=607$ vs. $n=244$ ). Despite these differences, the size of the metastatic foci correlated with NSLN involvement in both our and Gur et al.'s [12] study. The mean radius of the metastatic foci in our study was $4.6 \mathrm{~mm}$ (range $0.1-22 \mathrm{~mm}$ ) while it was $15 \mathrm{~mm}$ (range 2-41 $\mathrm{mm}$ ) in Gur et al.'s [12] study. To our knowledge, in the only study to test this model, Hidar et al. [17] reported a ROC value of 0.75 (95\% CI $0.63-0.88)$ for their patient population. The authors of this far smaller study $(\mathrm{n}=87)$ stated that they did not utilized the Turkish nomogram in a considerable number of patients thus lowering the reliability of the ROC results. In addition, Hidar et al. [17] reported that the Stanford model was the most efficient model for their patient group (ROC 0.76). Having covered a higher number of patients with racial similarity, we can state that ours is the most significant study to date validating Gur et al.'s [12] model.

The radius of the metastatic foci in the SLN and Cerb-2 positivity correlated with the likelihood of NSLN metastasis in our patient population. The predictive power of our formula was 0.722 . In that respect, it has similar power to the MSKCC nomogram. In reviewing the literature, one can see that the radius of the metastatic foci correlated with NSLN metastasis in the Turkish, Stanford, and Cambridge model. However, this is the first time that Cerb-2 is emerging as a predictive factor for NSLN metastasis. Cerb-2 is a well known negative prognostic factor in breast cancer; however, our knowledge about its effect on lymph node metastasis is limited. Only in 1 article by Mustac et al. [25] Cerb-2 status was found to correlate with the overall status of the axilla $(\mathrm{pN})$. In addition, the authors of the models that were used in our study did not state whether they tested Cerb-2 as a variable in their models. In the original article by Barranger et al. [9], a Cerb-2 positivity rate of $2.6 \%$ was reported for the entire study groups ( $25.9 \%$ in our patient population). This disparity could have resulted from differences in the evaluation methods.

The results of the Z0011 study by the American College of Surgeons Oncology Group, which was also cited in the original article of Van Zee [8], were published in 2010 [26]. This and similar studies stating inessentiality of AD can be predicted to lower the importance of nomograms. Despite the big uproar the study caused, some points need to be considered. Firstly, all patients enrolled in the Z0011 study had earlystage breast cancer (most having non-palpable tumors) and received adjuvant chemo- and radiotherapy. Another and 
more important point is that there is an inadequate number of prospective studies having validated the results. There will be a considerable number of patients beyond the coverage of this study (i.e. patients with total mastectomy and/or patients with micrometastases) who can be affected by high false negativity rates in frozen section analysis should the results be integrated in routine practice. Despite the nomograms being criticized for not having proper cut-off values, they continue to be useful tools facilitating decision-making by both patients and surgeons in today's breast cancer practice. However, although our nomogram showed promising results, it needs further validation in independent studies as well as cross validation with different patient populations, with the potential to yield deeper insights into the nature of nodal disease.

\section{Conclusion}

To date, no nomogram can predict SLN and NSLN involvement with $100 \%$ accuracy. Our study confirmed the MSKCC nomogram to be the most accurate. In addition, the discriminative value of our own unique predictivity formula was found to be similar to that of the MSKCC nomogram. Moreover, using only 2 patient-depended variables, our model facilitates clinical usage. To our knowledge, this is the second study in the literature to have validated the MHDF MF-08 nomogram. Likewise, our nomogram needs to be validated by future studies.

\section{Disclosure Statement}

The contributing authors have no conflict of interest.

\section{References}

1 Carter CL, Allen C, Henson DE: Relation of tumor size, lymph node status, and survival in 24,740 breast cancer cases. Cancer 1989;63:181187.

2 Dayangac M, Makay O, Yeniay L, Aynaci M, Kapkac M,Yilmaz R: Precipitating factors for lymphedema following surgical treatment of breast cancer: implications for patients undergoing axillary lymph node dissection. Breast J 2009;15:210 211.

3 Warmuth MA, Bowen G, Prosnitz LR, et al. Complications of axillary lymph node dissection for carcinoma of the breast: a report based on a patient survey. Cancer 1998;83:1362-1368.

4 Guiliano AE, Kirgan DM, Guenther JM, Morton DL: Lymphatic mapping and sentinel lymphadenectomy for breast cancer. Ann Surg 1994;220:391401.

$\checkmark 5$ Kamath VJ, Giuliano R, Dauway EL, et al: Characteristics of the sentinel lymph node in breast cancer predict further involvement of higherechelon nodes in the axilla: a study to evaluate the need for complete axillary dissection. Arch Surg 2001;136:688-692.

6 Sachdev U, Murphy K, Derzie A, et al.: Predictors of non-sentinel lymph node metastasis in breast cancer patients. Am J Surg 2002;183:213-217.

7 Turner RR, Chu KU, Hansen NM, Qi K, Botnick LE, Glass EC, et al.: Pahtologic features associated with nonsentinel lymph node metastases in patients with metastatic breast carcinoma in a sentinel lymph node. Cancer 2000;89:574-581.

8 Van Zee KJ, Manasseh DM, Bevilacqua JLB, Boolbol SK, Fey JV, Tan JV, et al.: A nomogram for predicting the likelihood of additiona nodal metastases in breast cancer patients with positive sentinel node biopsy. Ann Surg Oncol 2003;10:1140-1151.

9 Barranger E, Coutant C, Flahault A, Delpech Y, Darai E, Uzan S: An axilla scoring system to predict non-sentinel lymph node status in breast cancer patients with sentinel lymph node involvement. Breast Cancer Res Treat 2005;91:113-119.

- 10 Pal A, Provenzano E, Duffy SW, Pinder SE, Purushotham AD: A model for predicting non- sentinel lymph node metastatic disease when the sentinel lymph node is positive. Br J Surg 2008;95:302-309.

11 Kohrt HE, Olshen RA, Bermas HR, et al.; Bay Area SLN Study: New models and online calculator for predicting non-sentinel lymph node status in sentinel node positive breast cancer patients. BMC Cancer 2008;8:66.

12 Gur AS, Unal B, Ozbek U, Ozmen V, Aydogan F, Gokgoz S, Gulluoglu BM, et al.: Validation of breast cancer nomograms for predicting the nonsentinel lymph node metastases after a postive sentinel lymph node biopsy in a multi-center study. Eur J Surg Oncol 2010;36:30-35.

13 Cox CE, Pendas S, Cox JM, Joseph E, Shons AR, Yeatman T, et al.: Guidelines for sentinel node biopsy and a lymphatic mapping of patients with breast cancer. Ann Surg 1998;227:645-651.

14 Bolster MJ, Peer P, Bult P, Thunissen F, Schapers R, Meijer J, et al.: Risk factors for non-sentinel lymph node metastases in patients with breast cancer. The outcome of a multi-institutional study. Ann Surg Oncol 2006;14:181-189.

15 Rahusen FD, Torrenga H, van Diest PJ, Pijpers R, van der Wall E, Licht J, Meijer S: Predictive factors for metastatic involvement of nonsentinel nodes in patients with breast cancer. Arch Surg 2001;136:1059-1063.

16 Fleming FJ, Kavanagh D, Crotty TB, Quinn CM, McDermott EW, O'Higgins N, Hill AD: Factors affecting metastases to non-sentinel lymph nodes in breast cancer. J Clin Pathol 2004;57:73-76.

17 Hidar S, Harrabi I, Benregaya L, Fatnassi R, Khelifi A, Benabdelkader A, et al.: Validation of nomograms to predict the risk of non-sentinel lymph node metastases in North African Tunisian breast cancer patients with sentinel node involvement. Breast 2011;20:26-30.

18 Van la Parra RFD, Ernst MF, Bevilacqua JLB, Mol SJJ, van Zee KJ, Broekeman JM, Bosscha K: Validation of a nomogram to predict the risk of nonsentinel lymph node metastases in breast cancer patients with a positive sentinel node biopsy: validation of the MSKCC breast nomogram. Ann Surg Oncol 2009;16:1128-1135.
19 Bevilacqua JL, Kattan MW, Fey JV, Cody HS 3rd, Borgen PI, van Zee KJ: Doctor, what are my chances of having a positive sentinel node? A validated nomogram for risk estimation. J Clin Oncol 2007;25:3670-3679.

20 Dauphine CE, Haukoos JS, Vargas MP, Isaac NM, Khalkali I, Vargas HI: Evaluation of three scoring systems predicting non sentinel node metastasis in breast cancer patients with a positive sentinel node biopsy. Ann Surg Oncol 2007;14:1014-1019.

21 Zgajnar J, Perhavec A, Hocevar M, Podkrajsek M, Hertl K, Frkovic-Grazio S, et al.: Low performance of the MSKCC nomogram in preoperatively ultrasonically negative axillary lymph node in breast cancer patients. J Surg Oncol 2007;96:547e53.

22 Pal A, Provenzano E, Duffy SW, et al.: A model for predicting non-sentinel lymph node metastatic disease when the sentinel node is positive. $\mathrm{Br} \mathrm{J}$ Surg 2008;95:302-309.

23 D'Eredita G, Troilo VL, Giardina C, Napoli A, et al.: Sentinel lymph node micrometastasis and risk of non-sentinel lymph node metastasis: validation of two breast cancer nomograms. Clin Breast Cancer 2010;10:445-451.

24 Coutant C, Rouzier R, Fondriener E, Marchal F, Guillemin F, Seince N, Rodrigues A, Darai E, Uzan S, Barranger E: Validation of the Tenon breast cancer score for predicting non-sentinel lymph node status in breast cancer patients with sentinel lymph node metastasis: a prospective multicenter study. Breast Cancer Res Treat 2009;113:537-543.

25 Mustac E, Matusan-Ilijas K, Marijic B, Smokvina M, Jonjic N: Predicting the likelihood of additional nodal metastases in breast carcinoma patients with positive sentinel node biopsy. Int J Surg Pathol 2010;18;36-41.

26 Giuliano AE, McCall L, Beitsch P, Whitworth PW, Blumencranz P, Leitch AM, et al.: Locoregional recurrence after sentinel lymph node dissection with or without axillary dissection in patients with sentinel lymph node metastases: the American College of Surgeons Oncology Group Z0011 randomized trial. Ann Surg 2010;252:426-433. 